\title{
Numerical Simulation of Self-heating InGaP/GaAs Heterojunction Bipolar Transistors
}

\author{
Yiming $\mathrm{Li}^{1,2}$ and Kuen-Yu Huang ${ }^{3}$ \\ 1 Department of Computational Nanoelectronics, \\ National Nano Device Laboratories, Hsinchu 300, Taiwan \\ 2 Microelectronics and Information Systems Research Center, \\ National Chiao Tung University, Hsinchu 300, Taiwan \\ ymli@faculty.nctu.edu.tw \\ 3 Institute of Electronics, National Chiao Tung University, \\ Hsinchu 300, Taiwan
}

\begin{abstract}
We numerically simulate effects of the self-heating on the current-voltage characteristics of $\mathrm{InGaP} / \mathrm{GaAs}$ heterojunction bipolar transistors (HBTs). A set of coupled nonlinear ordinary differential equations (ODEs) of the equivalent circuit of HBT is formed and solved numerically in the large-signal time domain. We decouple the corresponding ODEs using the waveform relaxation method and solve them with the monotone iterative method. The temperature-dependent energy band gap, the current gain, the saturation current, and the thermal conductivity are considered in the model formulation. The power-added efficiency and the 1-dB compression point of a three-finger HBT are calculated. This approach successfully explores the self-heating and the thermal coupling phenomena of the three-finger transistors under high power and high frequency conditions. The numerical algorithm reported here can be incorporated into electronic computer-aided design software to simulate ultra-large scale integrated and radio frequency circuits.
\end{abstract}

\section{Introduction}

High power heterojunction bipolar transistors (HBTs) operated at micrometerand millimeter-wave frequencies have been of great interest; in particular, for the advanced wireless and fiber communication [1], [2], [3], [4], [5]. These transistors, fabricated for high power applications, usually have a structure of multiple fingers to spread the current and the dissipated heat. Therefore, effects of the self-heating and the thermal coupling among fingers is one of the important issues for design and fabrication of advanced radio frequency ( $\mathrm{RF}$ ) circuits. Electrical characteristics depend on the surrounding temperature, so the thermal effect significantly influences the linearity of operating transistors [6]. Therefore, a self-heating model should be considered when exploring power dissipations of $\mathrm{InGaP} / \mathrm{GaAs}$ devices and related RF circuits.

In this paper we examine effects of the self-heating on a three-finger In$\mathrm{GaP} / \mathrm{GaAs} \mathrm{HBT}$ operated at high frequency. Using the waveform relaxation 


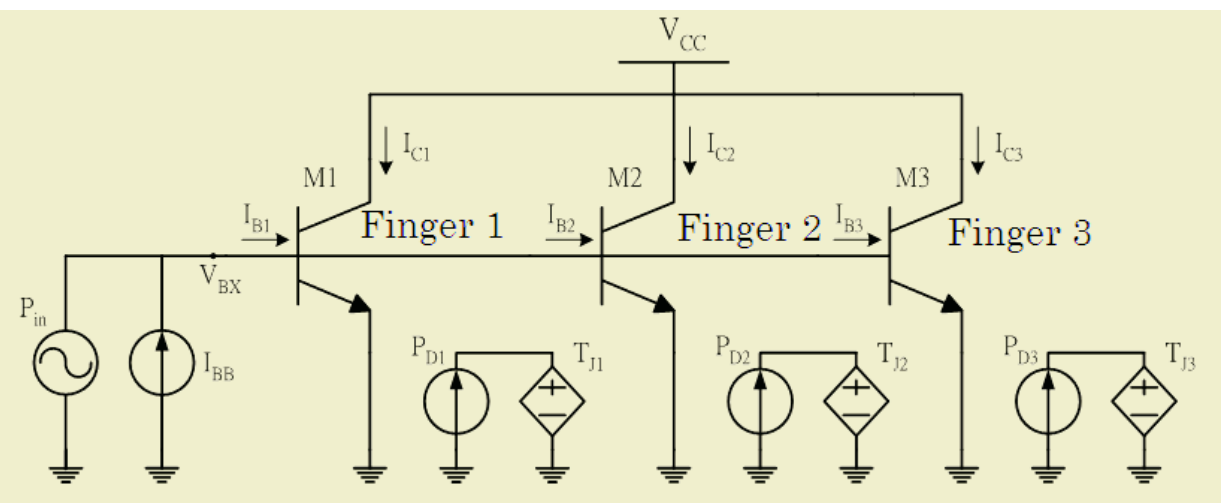

Fig. 1. An equivalent circuit of the simulated three-finger HBT under constant current and high frequency excitations

(WR), the monotone iterative (MI), and Runge-Kutta methods [7], [8], [9], [10], a set of nonlinear ordinary differential equations (ODEs) with thermal models is solved numerically in the time domain. The governing ODEs of the equivalent circuit of HBTs are formulated by the Kirchhoff's current law [11]. This solution technique has recently been developed by us for circuit simulations [7], [8], [9], [10]. The temperature-dependent physical quantities are modeled and several important engineering factors, such as the power-added efficiency and the 1-dB compression point of the simulated three-finger HBT are calculated. Our modeling and simulation successfully explores the self-heating and the thermal coupling phenomena of the studied three-finger transistors circuit operated under high power and high frequency conditions.

This paper is organized as follows. In Sec. 2, we state the physical model and numerical method. In Sec. 3, we present the simulation results. Finally, we draw conclusions.

\section{Self-heating Modeling and Numerical Method}

A multifinger HBT is formed by several sub-HBTs with their own collector and emitter, where their base is connected together. As shown in Fig. 1, an equivalent circuit with a thermal network of the three-finger HBT is studied in this work, where each finger is theoretically assumed to be identical. A thermal model that describes the relation between the power dissipation and the junction temperature is adopted. The electrical model of HBT considered in our simulation is based on the Gummel-Poon (GP) large signal model [7], [8], [9], [10]. For the thermal-electrical feedback mechanism, the temperature-dependant equations are introduced to the GP model

$$
E_{g}\left(T_{J}\right)=E_{g}\left(T_{A}\right)+\frac{E_{a} \cdot T_{A}^{2}}{T_{A}+E_{b}}+\frac{E_{a} \cdot T_{J}^{2}}{T_{J}+E_{b}}
$$




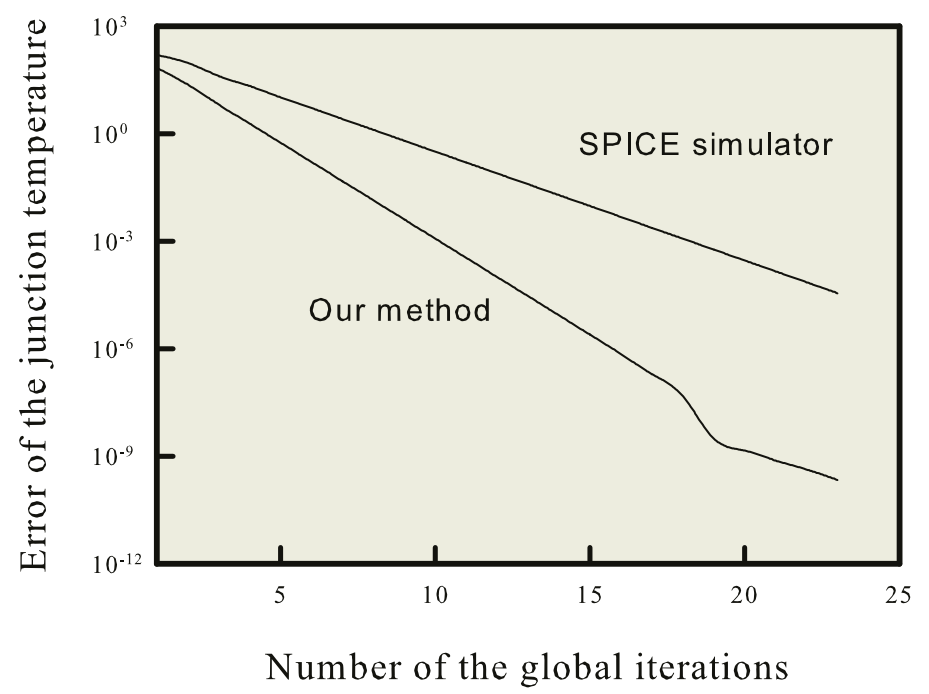

Fig. 2. A plot of the maximum norm error of the junction temperature versus the number of iterations of the WR loop

$$
\begin{gathered}
I S\left(T_{J}\right)=I S \cdot\left(\frac{T_{J}}{T_{A}}\right)^{X T I} \cdot \exp \left[\left(\frac{E_{g}\left(T_{A}\right)}{k \cdot T_{A}}\right)-\left(\frac{E_{g}\left(T_{J}\right)}{k \cdot T_{J}}\right)\right], \\
I S E\left(T_{J}\right)=I S E \cdot\left(\frac{T_{J}}{T_{A}}\right)^{\frac{X T I}{N E}-X T B} \cdot \exp \left[\left(\frac{E_{g}\left(T_{A}\right)}{N E \cdot k \cdot T_{A}}\right)-\left(\frac{E_{g}\left(T_{J}\right)}{N E \cdot k \cdot T_{J}}\right)\right], \\
I S C\left(T_{J}\right)=I S C \cdot\left(\frac{T_{J}}{T_{A}}\right)^{\frac{X T I}{N C}-X T B} \cdot \exp \left[\left(\frac{E_{g}\left(T_{A}\right)}{N C \cdot k \cdot T_{A}}\right)-\left(\frac{E_{g}\left(T_{J}\right)}{N C \cdot k \cdot T_{J}}\right)\right], \\
B F\left(T_{J}\right)=B F \cdot\left(\frac{T_{J}}{T_{A}}\right)^{X T B}, \\
B R\left(T_{J}\right)=B R \cdot\left(\frac{T_{J}}{T_{A}}\right)^{X T B},
\end{gathered}
$$

where $T_{J}$ and $T_{A}$ are the junction and the ambient temperature, respectively. We note that for high power devices, $T_{A}$ is the temperature on the back of the substrate. Above equations include the temperature dependance of energy band gap $\left(E_{g}\right)$, the saturation current $(I S)$, the collector and emitter leakage current $(I S C$ and $I S E)$, and the current gain $(B F$ and $B R)$. The thermal model expresses the relation between the power dissipation and the junction temperature. The junction temperature with considering the temperature-dependent thermal conductivity for the three-finger HBT is given by 


$$
\mathbf{T}_{J}=T_{A}\left\{1-\frac{(B B-1)}{T_{A}}\left[\mathbf{R}_{T H} \cdot \mathbf{P}_{D}\right]\right\}^{\frac{-1}{B B-1}}=\left[\begin{array}{c}
T_{J 1} \\
T_{J 2} \\
T_{J 3}
\end{array}\right]
$$

where $T_{J n}$ is the junction temperature of the $n^{t h}$ finger, $n=1,2,3 . \mathbf{R}_{T H} \cdot \mathbf{P}_{D}$ is given by

$$
\mathbf{R}_{T H} \cdot \mathbf{P}_{D}=\left[\begin{array}{lll}
R_{T 11} & R_{T 12} & R_{T 13} \\
R_{T 21} & R_{T 22} & R_{T 23} \\
R_{T 31} & R_{T 32} & R_{T 33}
\end{array}\right] \cdot\left[\begin{array}{c}
P_{D 1} \\
P_{D 2} \\
P_{D 3}
\end{array}\right]
$$

$R_{T n n}$ and $R_{T n m}$ denote the self-heating thermal resistance of the $n^{\text {th }}$ finger and the coupling thermal resistance which counts the coupled heat from the $m^{\text {th }}$ finger to the $n^{\text {th }}$ finger, respectively. Furthermore, the power dissipation of the $n^{\text {th }}$ finger is denoted by $P_{D n}$. Equivalent circuit of the three-finger HBT is shown in Fig. 1. The finger 1 of this HBT is represented by $M 1$, and $M 2$ and $M 3$ are the fingers 2 and 3 , respectively. $I_{B B}$ denotes the constant bias current at the base and $P_{i n}$ is the power of RF input signal. The behavior of Fingers 1 and 3 is the same for the identical fingers assumption. By considering the models above, the electrical and thermal feedback equations for the power HBTs are achieved and solved self-consistently in the time domain, where the temperature-dependent thermal conductivity is also included in our numerical solution.

The corresponding coupled nonlinear ODEs of the equivalent circuit of HBTs above is first decoupled using the WR method and then solved with the MI and Runge-Kutta methods. Compared with the conventional method, the Netwon's iteration method, used in the well-known SPICE circuit simulator [8], [9] our approach in solving the self-heating model is robust and cost-effective for the large-scale time domain circuit simulation of HBTs.

\section{Results and Discussion}

First of all, we verify the convergence of the solution method. The testing case is the device with the collector voltage $V_{C C}=5 \mathrm{~V}$, the input current bias $I_{B B}=$ $0.5 \mathrm{~mA}$, the frequency is centralized at $1.8 \mathrm{GHz}$, and the convergence criterion is that the maximum norm error of the output voltage is less than $10^{-11} \mathrm{~V}$. Figure 2 shows a plot of the maximum norm error of the junction temperature versus the number of iterations. Simulation with our method demonstrates better convergence property than the result of the SPICE circuit simulator [9]. However, the simulation with the SPICE circuit simulator takes more than 100 iterations to meet the specified stopping criterion. For each time step, the CPU time of our method is of the order of $10^{2}$ sec. running on a HP workstation XW 8000 . The convergence of SPICE circuit simulator depends upon the selection of initial guess and the time steps which complicates the solution procedure for practical engineering application. Our solution algorithm converges monotonically and the DC solutions, in general, are used as the starting solution. 


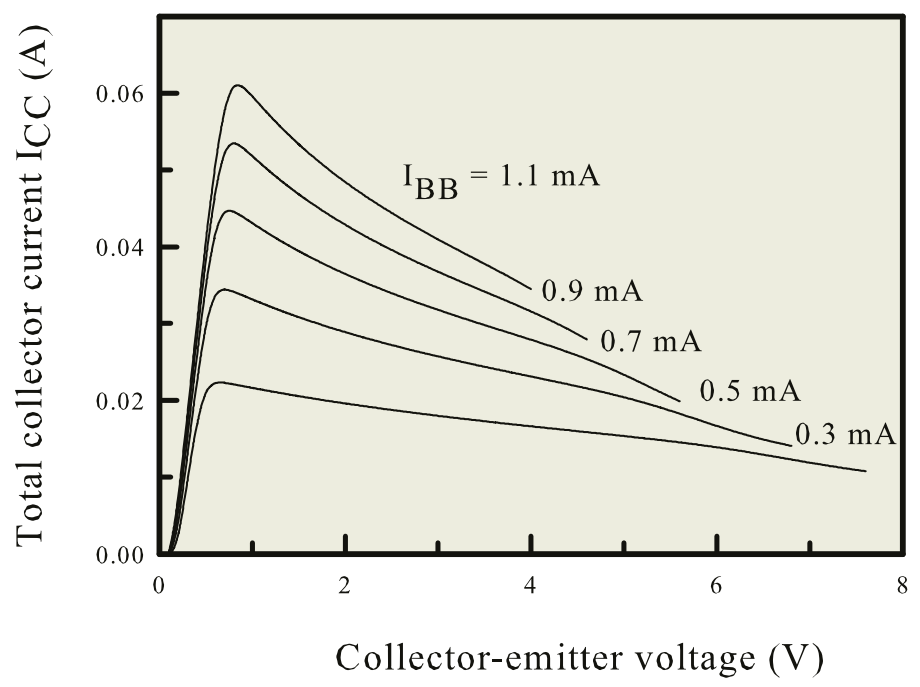

Fig. 3. The curves of $I_{C C}-V_{C E}$ with respect to different $I_{B B}$ for the simulated threefinger InGaP/GaAs HBT circuit. It is significantly different from the result without considering the self-heating [7], [8], [9]

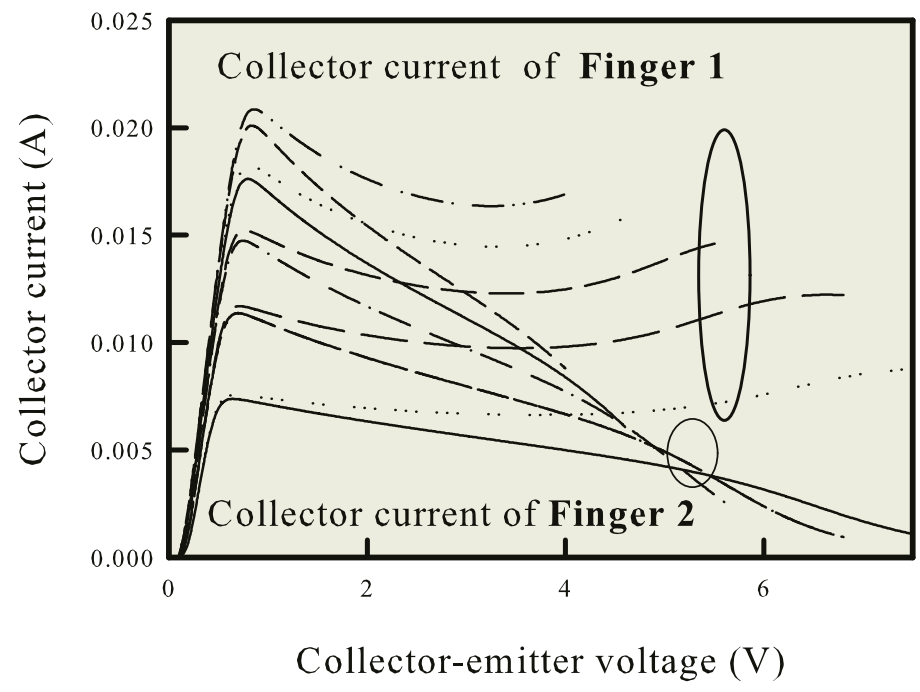

Fig. 4. The simulated $I_{C C}-V_{C E}$ curves with respect to different $I_{B B}$ for the fingers 1 and 2 of the HBT. The result of Finger 3 is omitted according to the property of symmetry

The simulated I-V characteristics of the three-finger InGaP/GaAs HBT circuit are shown in Figs. 3 and 4. Each curve in these figures represents the col- 


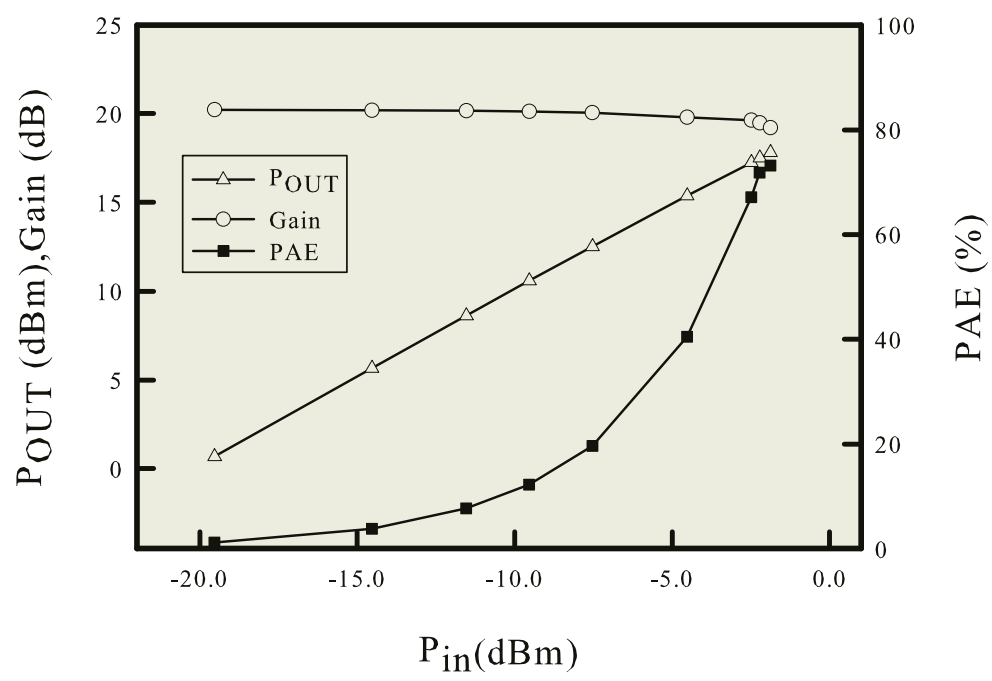

Fig. 5. The computed $P_{O U T}, \mathrm{PAE}$, and Gain versus $P_{i n}$

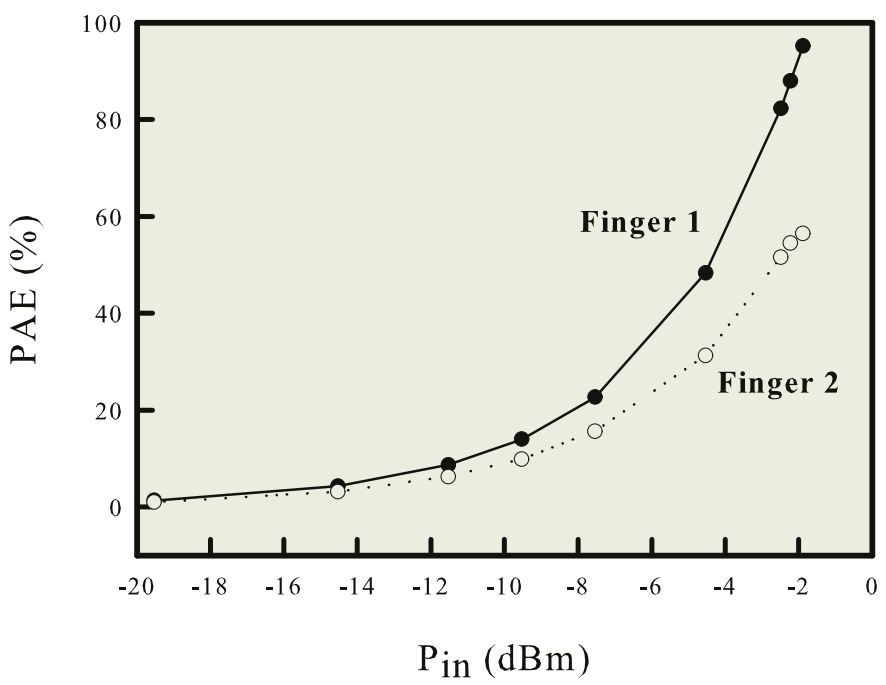

Fig. 6. Plots of the PAE of Fingers 1 and 2 versus the input power

lector current under constant $I_{B B}$. Due to the effect of self-heating, the total collector current decreases when the collector-emitter voltage increases, shown in Fig. 3. It results in a negative differential resistance region in the I-V characteristics and a collapse of current gain in the three-finger HBT circuit. As shown in Fig. 4, the collector current of the central finger (Finger 2) decreases rapidly, compared with the results of neighbor figures, such as Finger 1. It is due 
to the strongly coupled heat from its neighbor two fingers, Finger 1 and Finger 3. As the collector-emitter voltage increases even more $\left(V_{C C}>4 \mathrm{~V}\right)$, an abrupt reduction of the collector current of Finger 2 occurs. With the electrical and thermal interaction, our modeling and simulation can explore the phenomenon of collapse for the multifinger HBTs under high voltage and high current bias.

As shown in Fig. 5, it is the calculated output power $\left(P_{O U T}\right)$, the poweradded efficiency (PAE), and the power gain (Gain) versus the different values of the input power $\left(P_{i n}\right)$. The input excitation is a single tone signal at $1.8 \mathrm{GHz}$. The bias condition of this single tone simulation is with $V_{C C}=3.6 \mathrm{~V}$ and $I_{B B}=$ $0.6 \mathrm{~mA}$. In this simulation, we have taken the effect of heating of the input high frequency signal into consideration. It is found that, shown in Fig. 5, the Gain and PAE degrade as $P_{i n}$ increases, and the $1-d B$ compression point $\left(P_{1-d B}\right)$ is $-2.45 \mathrm{dBm}$. The effect of thermal coupling among fingers also influences the performance of the three-finger device structure. As shown in Fig. 6, the PAE of the central finger (Finger 2) is reduced and degraded when $P_{i n}>-3 \mathrm{dBm}$. In the meanwhile, the PAE of the neighbor finger (Finger 1) still increases as $P_{\text {in }}$ increases. This phenomenon illustrates that the performance degradation of the whole transistor is mainly dominated by the hotter central finger.

\section{Conclusions}

In this paper, we have numerically solved a set of nonlinear and self-heating ODEs for exploring the electrical characteristics of InGaP/GaAs device. The solution approach is mainly based on the WR and the MI methods. Compared with the well-known SPICE circuit simulator, our approach has successfully shown its robustness. Different electrical characteristics have been calculated to examine effects of self-heating on the simulated three-finger HBT circuit. We believe that the solution method will benefit the community of electronic computer-aided design; in particular, for modern RF circuit simulation. To perform optimal designs for specified circuits, we are currently implementing intelligent algorithms with the developed method.

\section{Acknowledgments}

This work is supported in part by the National Science Council (NSC) of TAIWAN under contracts NSC-93-2215-E-429-008 and NSC 93-2752-E-009-002-PAE, and the grant of the Ministry of Economic Affairs, TAIWAN under contracts No. 92-EC-17-A-07-S1-0011 and No. 93-EC-17-A-07-S1-0011.

\section{References}

1. Yanagihara, M., Sakai, H., Ota, Y., Tamura, A: High fmax AlGaAs/GaAs HBT with L-shaped base electrode and its application to $50 \mathrm{GHz}$ amplifier. Solid-State Electron. 41 (1997) 1615-1620 
2. Oka, T., Hirata, K., Suzuki, H., Ouchi, K., Uchiyama, H., Taniguchi, T., Mochizuki, K., Nakamura, T: High-speed small-scale InGaP/GaAs HBT technology and its application to integrated circuits. IEEE Trans. Electron Devices. 48 (2001) 26252630

3. Troyanovsky, B., Yu, Z., Dutton, R. W.: Physics-based simulation of nonlinear distortion in semiconductor devices using the harmonic balance method. Comput. Methods Appl. Mech. Engrg. 181 (2000) 467-482

4. Zhu, Y., Twynam, J. K., Yagura, M., Hasegawa, M., Hasegawa, T., Eguchi, Y., Amano, Y., Suematsu, E., Sakuno, K., Matsumoto, N., Sato, H., Hashizume, N.: Self-heating effect compensation in HBTs and its analysis and simulation. IEEE Trans. Electron Devices. 48 (2001) 2640-2646

5. Heckmann, S., Sommet, R., Nebus, J.-M., Jacquet, J.-C., Floriot, D., Auxemery, P., Quere, R.: Characterization and modeling of bias dependent breakdown and self-heating in GaInP/GaAs power HBT to improve high power amplifier design. IEEE Trans. Microwave Theory and Techniques 50 (2002) 2811-2819

6. Park, H.-M., Hong, S.: A novel temperature-dependent large-signal model of heterojunction bipolar transistor with a unified approach for self-heating and ambient temperature effects. IEEE Trans. Electron Devices. 49 (2002) 2099-2106

7. Li, Y., Cho, Y.-Y., Wang, C.-S., Hung, K.-Y.: A Genetic Algorithm Approach to InGaP/GaAs HBT Parameters Extraction and RF Characterization. Jpn. J. Appl. Phys. 42 (2003) 2371-2374

8. Huang, K.-Y., Li, Y., Lee, C.-P.: A Time Domain Approach to Simulation and Characterization of RF HBT Two-Tone Intermodulation Distortion. IEEE Trans. Microwave Theory and Techniques. 51 (2003) 2055-2062

9. Li, Y., Kuang, K.-Y.: A Novel Numerical Approach to Heterojunction Bipolar Transistor Circuit Simulation. Comput. Phys. Commun. 152 (2003) 307-316

10. Li, Y.: A Monotone Iterative Method for Bipolar Junction Transistor Circuit Simulation. WSEAS Trans. Mathematics. 1 (2002) 159-164

11. Liu, W.: Handbook of III-V Heterojunction Bipolar Transistor. John Wiley \& Sons (1998) 